Larry Joel Goldstein

Nagoya Math. J.

Vol. 53 (1974), 171-187

\title{
DEDEKIND SUMS FOR A FUCHSIAN GROUP, II
}

\author{
LARRY JOEL GOLDSTEIN ${ }^{1}$
}

\section{Introduction}

In [1] we derived a generalization of Kronecker's first limit formula. Our generalization was a limit formula for the Eisenstein series for an arbitrary cusp of a Fuchsian group $\Gamma$ of the first kind operating on the complex upper half-plane $\boldsymbol{H}$. In that work, we introduced Dedekind sums associated to the principal congruence subgroups $\Gamma(N)$ of the elliptic modular group. The work of our preceding paper suggests a natural question: Is there a generalization of Kronecker's second limit formula to the setting of a general Fuchsian group of the first kind? The answer to this question is the subject of this paper.

In our preceding work, we viewed the Eisenstein series as a generalization of the classical series

$$
\sum_{(m, n) \neq(0,0)} \frac{y^{s}}{|m+n z|^{2 s}}, \quad z=x+i y, \quad y>0, \quad \operatorname{Re}(s)>1 .
$$

In order to generalize Kronecker's second limit formula, it is necessary to find an intrinsic interpretation for the classical series

$$
\begin{gathered}
E^{*}(z, s \mid u, v)=y^{s} \sum_{(m, n) \neq(0,0)} \frac{e^{2 \pi i(m u+n v)}}{|m+n z|^{2 s}}, \\
u, v \text { real, } z=x+i y, y>0, \operatorname{Re}(s)>1
\end{gathered}
$$

in terms of the group theory of our Fuchsian group $\Gamma$. The behavior of the generalized function near $s=1$ gives rise to a generalization of Kronecker's second limit formula. For our purposes, the series $E^{*}(z, s \mid u, v)$ is not the natural object to generalize, but rather the series

$$
E(z, s \mid u, v)=y^{s} \sum_{\substack{(m, n) \neq(0,0) \\(m, n)=1}} \frac{e^{2 \pi i(m u+n v)}}{|m+n z|^{2 s}} .
$$

Received August 29, 1973.

1 Research supported by NSF Grant GP-31820X. 
We can compute $E^{*}(z, s \mid u, v)$ from $E(z, s \mid u, v)$ via

$$
E^{*}(z, s \mid u, v)=\sum_{k=1}^{\infty} \frac{1}{k^{2 s}} E(z, s \mid k u, k v)
$$

so that any limiting behavior of $E(z, s \mid u, v)$ at $s=1$ can easily be transcribed to $E^{*}(z, s \mid u, v)$.

The series $E(z, s \mid u, v)$ will be a typical example of what we call a generalized Eisenstein series, with the series (*) being the generalized Eisenstein series for the Fuchsian group $\Gamma=S L(2, Z)$. In this paper, we will define the generalized Eisenstein series for any Fuchsian group of the first kind which is contained in $S L(2, Z)$ and for which $-1 \in \Gamma$. (The latter hypothesis is merely for the sake of convenience.) We will study the analytic continuability of our generalized Eisenstein series past the line $\operatorname{Re}(s)=1$. From our analytic continuation, we will deduce a Kronecker limit formula for our generalized Eisenstein series and will study the properties of the analogues of the classical $\eta$-function which appear in our limit formula.

\section{Definition of the Generalized Eisenstein Series}

Throughout this paper, let $\Gamma$ be a Fuchsian group of the first kind, i.e. a discrete subgroup of $S L(2, \boldsymbol{R})$ with a fundamental domain $D$ of finite invariant volume. Further, we will assume that $\Gamma$ has at least one cusp. For the sake of convenience, let us assume that $-1 \in \Gamma$. Finally, let us assume that $\infty$ is a cusp of $\Gamma$. We will carry out our definition of the generalized Eisenstein series for the cusp at infinity only. It will be clear from our reasoning how to define Eisenstein series for any other cusp. In order to completely study our generalized Eisenstein series, it will be necessary for us to assume that $\Gamma \subseteq S L(2, Z)$, but this hypothesis is not necessary in this section.

Throughont $z=x+i y$ will denote a point of the complex upper half-plane $\boldsymbol{H}$ and we will set $y=y(z)$. We will denote by $\Gamma_{\infty}$ the stabilizer of $\infty$ in $\Gamma$. Then, since $-1 \in \Gamma$,

$$
\Gamma_{\infty}=\left\{ \pm\left(\begin{array}{cc}
1 & n \alpha \\
0 & 1
\end{array}\right) \mid n \in Z\right\}
$$

for some $\alpha>0$. Let $\sigma_{\infty} \in S L(2, R)$ be such that $\sigma_{\infty}^{-1} \Gamma_{\infty} \sigma_{\infty}=\left\{ \pm\left(\begin{array}{ll}1 & n \\ 0 & 1\end{array}\right) \mid n \in Z\right\}$, 
e.g. $\sigma_{\infty}=\left(\begin{array}{cc}\sqrt{\alpha} & 0 \\ 0 & \sqrt{\alpha}^{-1}\end{array}\right)$.

Let us fix $u, v \in \boldsymbol{R}$ and let us consider the series

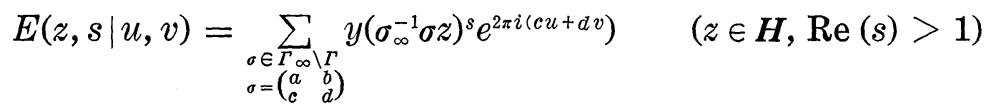

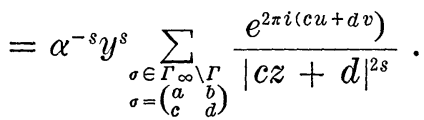

It is immediate from the definition that this series does not depend on the choice of the coset representatives of $\sigma \in \Gamma_{\infty} \backslash \Gamma$.

Note that if $(u, v)=(0,0)$, then we see that

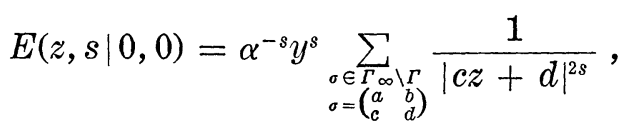

which is the usual Eisenstein series associated to the cusp $\infty$ of $\Gamma$ (See [2].). Moreover, by comparing the terms of $E(z, s \mid u, v)$ with the corresponding terms of the usual Eisenstein series, we deduce that the series for $E(z, s \mid u, v)$ converges absolutely and uniformly on compact subsets of the half-plane $\operatorname{Re}(s)>1$. In particular, $E(z, s \mid u, v)$ is analytic for $\operatorname{Re}(s)>1$.

Let $D=y^{-2}\left(\partial^{2} / \partial x^{2}+\partial^{2} / \partial y^{2}\right)$ denote the Laplace-Beltrami operator for the Riemannian symmetric space $\boldsymbol{H}$ with respect to the action of $S L(2, \boldsymbol{R})$. Then $D$ is an invariant differential operator on $\boldsymbol{H}$. Moreover, since

$$
D y^{s}=s(s-1) y^{s},
$$

the invariance of $D$ implies that

$$
D E(z, s \mid u, v)=s(s-1) E(s, z \mid u, v) .
$$

Thus, $E(z, s \mid u, v)$ is an eigenfunction of $D$ corresponding to the eigenvalue $\lambda_{s}=s(s-1)$. In particular, $E(z, s \mid u, v)$ is a real-analytic function of $z$.

An elementary combinatorial argument shows that if $\sigma=\left(\begin{array}{ll}a & b \\ c & d\end{array}\right) \in \Gamma$, then

$$
E(\sigma z, s \mid a u+b v, c u+d v)=E(z, s \mid u, v) .
$$

The main problem treated in this paper is the analytic continuability 
of $E(z, s \mid u, v)$ beyond the half-plane $\operatorname{Re}(s)>1$.

Let us first get an explicit set of representatives for the cosets $\Gamma_{\infty} \backslash \Gamma$. First of all, it is trivial to see that $\Gamma_{\infty} \sigma=\Gamma_{\infty} \sigma^{\prime}\left(\sigma, \sigma^{\prime} \in \Gamma\right) \Leftrightarrow \sigma$ and $\sigma^{\prime}$ have the same second row. Next, note that

$$
\left(\begin{array}{ll}
a & b \\
c & d
\end{array}\right)\left(\begin{array}{cc}
1 & n \alpha \\
0 & 1
\end{array}\right)=\left(\begin{array}{cc}
a & n \alpha a+b \\
c & n \alpha c+d
\end{array}\right)
$$

Therefore, as a set of coset representatives of $\Gamma_{\infty} \backslash \Gamma$, we may take the set

$\left\{\left(\begin{array}{ll}1 & 0 \\ 0 & 1\end{array}\right)\right\} \cup\left\{\sigma r \mid \sigma=\left(\begin{array}{ll}* & * \\ c & d\end{array}\right) \in \Gamma \begin{array}{l}\text { runs over a set representing the second } \\ \text { rows of elements in } \Gamma, c>0, d \text { taken } \\ \text { only } \bmod c \alpha, r \in \Gamma_{\infty} .\end{array}\right\}$

Therefore, we may write

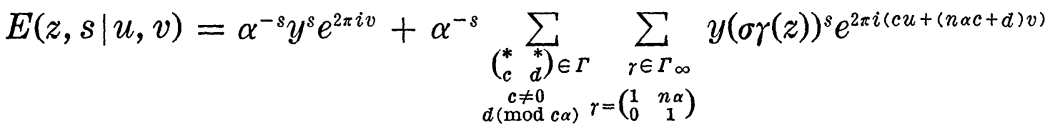

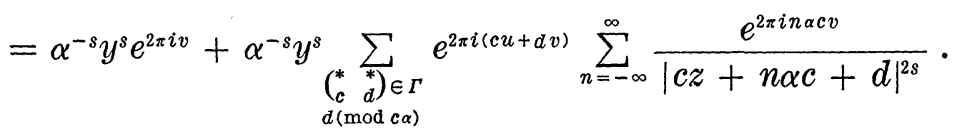

Let us denote

$$
I_{c, d}(z, s, v)=\sum_{n=-\infty}^{\infty} \frac{e^{2 \pi i n \alpha c v}}{|c z+n \alpha c+d|^{2 s}}
$$

Then

$$
E(z, s \mid u, v)=\alpha^{-s} y^{s} e^{2 \pi i v}+\alpha^{-s} y^{s} \sum_{\substack{* \\ c \\ d \\ d>0 \\ d(\bmod c \alpha)}} e^{2 \pi i(c u+d v)} I_{c, d}(z, s, v)
$$

By applying the Poisson summation formula to the sum (3), we derive

$$
\begin{aligned}
I_{c, d}(z, s, v) & =\sum_{m=-\infty}^{\infty} \int_{-\infty}^{\infty} \frac{e^{2 \pi i w(m+\alpha c v)}}{|c z+d+w \alpha c|^{2 s}} \\
& =|c|^{-2 s} \alpha^{-1} y^{1-2 s} \sum_{m=-\infty}^{\infty} e^{-2 \pi i(c x+d)(m+\alpha c v) / \alpha c} \int_{-\infty}^{\infty} \frac{e^{2 \pi i y w(m+\alpha c v) / \alpha}}{\left(w^{2}+1\right)^{s}} d w,
\end{aligned}
$$

where $z=x+i y$. It is well-known that for $u \neq 0, u \in R$, we have

$$
\int_{-\infty}^{\infty} \frac{e^{2 \pi i u t}}{\left(1+t^{2}\right)^{s}} d t=2 \pi^{s}|u|^{s-1 / 2} \Gamma(s)^{-1} K_{s-1 / 2}(2 \pi|u|),
$$


where $K_{s}(u)$ is the modified Bessel function defined by

$$
K_{s}(z)=\frac{\pi}{2} \frac{I_{-s}(z)-I_{s}(z)}{\sin (s \pi)}
$$

where

$$
I_{s}(z)=\sum_{m=0}^{\infty} \frac{(z / 2)^{s+m}}{m ! \Gamma(s+m+1)} .
$$

Moreover,

$$
\int_{-\infty}^{\infty} \frac{d t}{\left(1+t^{2}\right)^{s}}=\sqrt{\pi} \frac{\Gamma\left(s-\frac{1}{2}\right)}{\Gamma(s)} .
$$

Therefore, by (6), we have

$$
\begin{aligned}
I_{c, d}(z, s, v)= & 2 \pi^{s}|c|^{-2 s} \alpha^{-1} y^{1 / 2-s} \Gamma(s)^{-1} \sum_{\substack{m=-\infty \\
m \neq \alpha v c}}^{\infty} e^{-2 \pi i(c x+d)(-m+\alpha c v) / \alpha c} \\
& \cdot \alpha^{1 / 2-s}|-m+\alpha c v|^{s-1 / 2} K_{s-1 / 2}\left(\frac{2 \pi}{\alpha}|y(-m+\alpha c v)|\right) \\
& +E(\alpha c b) \sqrt{\pi} \alpha^{-1}|c|^{-2 s} y^{1-2 s} \frac{\Gamma\left(s-\frac{1}{2}\right)}{\Gamma(s)},
\end{aligned}
$$

where

$$
E(x)=\begin{array}{ll}
0 & \text { if } x \text { is not an integer } \\
1 & \text { if } x \text { in an integer. }
\end{array}
$$

Thus, by (4) and an elementary computation, we deduce the following expansion of our generalized Einstein series:

$$
\begin{aligned}
E\left(\sigma_{\infty} z, s \mid u, v\right)= & y^{s} e^{2 \pi i v}+\frac{2 \pi \sqrt{y}}{\Gamma(s)} \sum_{\substack{m=-\infty \\
m \neq 0}}^{\infty} a_{m}(s, z \mid u, v) e^{2 \pi i m x} \\
& +\sqrt{\pi} y^{1-s} \frac{\Gamma\left(s-\frac{1}{2}\right)}{\Gamma(s)} \phi(s \mid u, v), \quad(\operatorname{Re}(s)>1, z \in \boldsymbol{H}),
\end{aligned}
$$

where

$$
\begin{aligned}
a_{m}(s, z \mid u, v)= & \sum_{\substack{\left(\begin{array}{l}
* \\
c \\
d
\end{array}\right) \in \Gamma \\
d>0 \\
d(\bmod c \alpha) \\
v v c \neq m}} e^{2 \pi i(c u-\alpha c x v+d m / \alpha c)} \\
& \cdot|m-\alpha c v|^{s-1 / 2} K_{s-1 / 2}(2 \pi y|m-\alpha c v|)
\end{aligned}
$$

and 


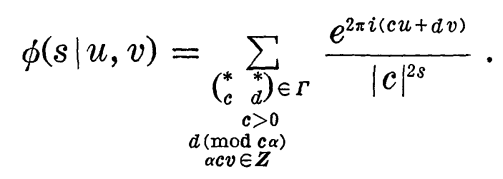

The formula (6) is the analogue in our setting of the Fourier series expansion of the usual Eisenstein series about the cusp at $\infty$. Note, however, the following remarks:

Remark 1: $E(z, s \mid u, v)$ is not usually periodic in $z$. This is reflected in the fact that the coefficient $a_{m}(s, z \mid u, v)$ depends on $x$. Note, however, that if $v=0$, then this dependence vanishes and $E(z, s \mid u, 0)$ is periodic in $z$ with period 1 . Note, also, that if $v$ is rational, say $v=$ $h / k$ and if $\Gamma \subseteq S L(2, Z)$, then $E(z, s \mid u, h / k)$ is periodic in $z$ with period $k$. In each of these two cases, the expansion (6) is the Fourier series which exhibits the periodicity.

Remark 2: The generalized Dirichlet series $\phi(s \mid u, v)$ is the precise generalization of the Dirichlet series which appears in the constant term of the Fourier expansion of the usual Eisenstein series. Note that in the expansion (6), we have isolated the third term which corresponds to the constant term in the usual theory.

\section{The Analytic Continuation of $E(z, s \mid u, v)$ for $\operatorname{Re}(s)>\frac{1}{2}$}

By using the same estimates as used to develop the analytic continuation of the usual Eisenstein series, we see that the series

$$
\frac{2 \pi^{s} \sqrt{y}}{\alpha \Gamma(s)} \sum_{\substack{m=-\infty \\ m \neq 0}}^{\infty} a_{m}(s, z \mid u, v) e^{2 \pi i m x / \alpha}
$$

converges absolutely and uniformly for $\operatorname{Re}(s)>\frac{1}{2}$. Therefore, in order to analytically continue $E(z, s \mid u, v)$ for $\operatorname{Re}(s)>\frac{1}{2}$, it suffices to analytically continue $\phi(s \mid u, v)$. By the classical theory of Eisenstein series, $\Phi(s \mid 0,0)$ can be analytically continued to a meromorphic function for $\operatorname{Re}(s)>\frac{1}{2}$, whose only poles satisfy $0<s \leq 1$. For $(u, v) \neq(0,0)$, we have not been able to establish such a general result. However, we can be specific if $u$ and $v$ are both rational. Namely, we will prove

THEOREM 3-1: Assume $u$ and $v$ are rational and that $\Gamma \subseteq S L(2, Z)$. The series $\phi(s \mid u, v)$ can be analytically continued to a meromorphic function for $\operatorname{Re}(s)>1-\varepsilon$ for some $\varepsilon>0$, such that the only poles of $\phi(s \mid u, v)$ 
in the half-plane are on the real axis. Moreover, at $s=1$, there is at most a simple pole.

Our proof of Theorem 3-1 is a reworking of the proof of the analytic continuability of the Eisenstein series given in [2]. Since the calculations are somewhat complicated, we will give fairly complete proofs where the details differ substantially from [2]. Our main hypotheses, namely that $\Gamma \subseteq S L(2, Z)$ and $u$ and $v$ are rational are already strongly utilized in the following lemma.

LEMMA 3-2: Assume that $\Gamma \subseteq S L(2, Z)$ and that $u$ and $v$ are rational. Let $f$ be a common denominator for $u$ and $v$ and let $\Gamma^{\prime}=\Gamma(f) \cap \Gamma$, where $\Gamma(f)=$ the principal congruence subgroup of $S L(2, Z)$ of level $f$. Then $E(z, s \mid u, v)$ is an automorphic function for $\Gamma^{\prime}$.

Proof: If $\sigma^{\prime}=\left(\begin{array}{ll}a^{\prime} & b^{\prime} \\ c^{\prime} & d^{\prime}\end{array}\right) \in \Gamma^{\prime}$, then $a^{\prime} \equiv d^{\prime} \equiv 1(\bmod f)$ and $b \equiv c \equiv 0$ $(\bmod f)$, so that

$$
E\left(\sigma^{\prime} z, s \mid \alpha^{\prime} u+b^{\prime} v, c^{\prime} u+d^{\prime} v\right)=E\left(\sigma^{\prime} z, s \mid u, v\right),
$$

as a simple computation shows. But then by (2), we have

$$
E\left(\sigma^{\prime} z, s \mid u, v\right)=E(z, s \mid u, v)
$$

whence the lemma.

Our proof of Theorem 3-1 will proceed in three steps. First, we will analytically continue $\phi(s \mid u, v)$ into the region $\operatorname{Re}(s)>\frac{1}{2}, s \notin\left(\frac{1}{2}, 1\right]$. Next, we will show how to extend the continuation to the segment $\left(\frac{1}{2}, 1\right]$. Finally, we will study the behavior at $s=1$.

Let us recall some preliminaries from [2]. Let $\psi(z)$ be a function on the upper half-plane such that $\psi(z+x)=\psi(z)$ for all $x \in \boldsymbol{R}$. Then the Mellin transform $L_{\psi}(s)$ of $\psi$ is defined by the formula

$$
L_{\psi}(s)=\int_{0}^{\infty} \psi(i y) y^{-s} \frac{d y}{y},
$$

and the inverse Mellin transform is given by

$$
\psi(i y)=\frac{1}{2 \pi i} \int_{\sigma-i \infty}^{\sigma+i \infty} L_{\psi}(s) y^{s} d s
$$

These formulas hold, for example, when $\psi(i y)=O\left(y^{-A}\right)(y \rightarrow \infty)$ for all 
$A>0$, and $\psi(i y)=O\left(y^{A}\right)(y \rightarrow 0)$ for all $A>0$. In this case, $\sigma$ may be an arbitrary real number. Whenever we consider functions $\psi$, they will be assumed to satisfy these conditions.

Let us associate to $\psi$ as above a generalized $\theta$-series $\theta_{\psi}(z \mid u, v)$ defined by

$$
\theta_{\psi}(z \mid u, v)=\sum_{\substack{\sigma \in \Gamma \infty \backslash \Gamma \\
\sigma=\left(\begin{array}{cc}
a & b \\
c & b
\end{array}\right)}} \psi(\sigma z) e^{2 \pi i(c u+d v)}
$$

By using the same reasoning as used in the proof of Lemma 3-1, we see that $\theta_{\psi}(z \mid u, v)$ is an automorphic function for the group $\Gamma^{\prime}=\Gamma \cap \Gamma_{f}$.

Let us consider the Hilbert space $\mathscr{H}=L^{2}\left(\boldsymbol{H} / \Gamma^{\prime}\right)$ with respect to the inner product

$$
(f, g)=\int_{\mathscr{Q}} f(z) \overline{g(z)} d z
$$

where $\mathscr{D}$ is a fundamental domain for $\Gamma^{\prime}$ and where $d z=d x d y / y^{2}, z=$ $x+i y$. By using the same reasoning as used in [2], it is easy to see that $\theta_{\psi}(z \mid u, v) \in \mathscr{H}$. Moreover, by using exactly the same reasoning as used in [2, p. 24], we derive the following inner product formula:

Let $\Gamma_{\infty}^{\prime}=$ the stabilizer of $\infty$ in $\Gamma^{\prime}$. Then $\Gamma_{\infty}^{\prime} \subseteq \Gamma_{\infty}$, so that

$$
\Gamma_{\infty}^{\prime}=\left\{ \pm\left(\begin{array}{cc}
1 & n \beta \\
0 & 1
\end{array}\right) \mid n \in Z\right\}
$$

for some $\beta>0$ such that $\beta / \alpha \in Z$. Then, we have

$$
\begin{aligned}
\left(\theta_{\psi}, \theta_{\psi^{\prime}}\right)= & \frac{\beta}{2 \pi i} e^{2 \pi i v} \int_{\sigma-i \infty}^{\sigma+i \infty} L_{\psi}(1-s) L_{\psi^{\prime}}(s) \\
& +\frac{\beta}{2 \pi i \alpha} \int_{\sigma-i \infty}^{\sigma+i \infty} L_{\psi}(s) L_{\psi^{\prime}}(s) \phi^{*}(s \mid u, v) d s,
\end{aligned}
$$

where $\phi^{*}(s \mid u, v)=\frac{\sqrt{\pi}}{\alpha} y^{1-s} \frac{\Gamma\left(s-\frac{1}{2}\right)}{\Gamma(s)} \phi(s \mid u, v)$.

Let us now take for $\psi$ the function such that

$$
\psi(y)=\frac{1}{2 \pi i} \int_{\sigma-i \infty}^{\sigma+i \infty} e^{s} y^{s} d s,
$$

and let, for $\operatorname{Re}(\zeta)>1$, us set

$$
\psi_{\zeta}(y)=\frac{1}{2 \pi i} \int_{\sigma-i \infty}^{\sigma+i \infty} \frac{e^{s^{2}}}{(s-\zeta)(s-1+\zeta)} y^{s} d s,
$$


where in the latter integral we choose $\sigma$ so that $\operatorname{Re}(1-\zeta)<\sigma<\operatorname{Re}(\zeta)$. These functions satisfy the growth conditions above. Let us write $\theta(z \mid u, v)$ instead of $\theta_{\psi}(z \mid u, v)$ and $\theta_{\zeta}(z \mid u, v)$ instead of $\theta_{\psi \zeta}(z \mid u, v)$. A simple computation shows that for $\sigma>1$, we have

$$
\psi_{\zeta}(z \mid u, v)=\frac{1}{2 \pi i} \int_{\sigma-i \infty}^{\sigma+i \infty} \frac{e^{s^{2}}}{(s-\zeta)(s-1+\zeta)} E(z, s \mid u, v) d s
$$

Therefore, if $D=y^{-2}\left(\partial^{2} / \partial x^{2}+\partial^{2} / \partial y^{2}\right)$ denotes the Laplacian, then

$$
(D-\zeta(\zeta-1) I) \theta_{\zeta}(z \mid u, v)=\theta(z),
$$

where $I$ denotes the identity operator of $\mathscr{H}$. Thus, if $\lambda$ is a complex number which is not real, then $D+\lambda$ has an inverse $R_{\lambda}$ which is a closed linear operator on $\mathscr{H}$ and

$$
\theta_{\zeta}(z \mid u, v)=R_{\lambda} \theta(z \mid u, v),
$$

with $\lambda=\zeta(\zeta-1)$. Moreover, for $\lambda$ not contained in the spectrum of $D$, we see that $\left(\theta, R_{\lambda} \theta\right)$ is an analytic function of $\lambda$. Therefore, $\left(\theta, \bar{\theta}_{\zeta}\right)$ is an analytic function of $\zeta$ for $\zeta$ non-real. Moreover, by the inner product formula (9), we have for $\sigma>1$ that

$$
\begin{aligned}
\left(\theta, \bar{\theta}_{\zeta}\right)= & \frac{\beta}{2 \pi i} e^{2 \pi i v} \int_{\sigma-i \infty}^{\sigma+i \infty} \frac{e^{(1-s)^{2}+s^{2}}}{(s-\zeta)(s-1+\zeta)} d s \\
& +\frac{\beta}{2 \pi i \alpha} \int_{\sigma-i \infty}^{\sigma+i \infty} \frac{e^{2 s^{2}} \phi(s \mid u, v)}{(s-\zeta)(s-1+\zeta)} d s .
\end{aligned}
$$

By shifting the line of integration to the line $\operatorname{Re}(s)=\sigma_{1}$, where $\sigma_{1}>\operatorname{Re}(\zeta)$, we see that

$$
\begin{aligned}
\left(\theta, \bar{\theta}_{\zeta}\right)= & \frac{\beta}{2 \pi i} e^{2 \pi i v} \int_{\sigma_{1}-i \infty}^{\sigma_{1}+i \infty} \frac{e^{(1-s)^{2}+s^{2}}}{(s-\zeta)(s-1+\zeta)} d s \\
& +\frac{\beta}{2 \pi i \alpha} \int_{\sigma_{1}-i \infty}^{\sigma_{1}+i \infty} \frac{e^{2 s} \phi^{*}(s \mid u, v)}{(s-\zeta)(s-1+\zeta)} d s \\
& -\frac{\beta}{\alpha} \frac{e^{2 \zeta^{2}}}{2 \zeta-1} \phi^{*}(\zeta \mid u, v)-\beta e^{2 \pi i v} \frac{e^{(1-\zeta)^{2}+\zeta^{2}}}{2 \zeta-1}
\end{aligned}
$$

From equation (10), we immediately deduce the following result:

Proposition 3-3: $\phi(s \mid u, v)$ may be analytically continued to a holomorphic function in the region $\operatorname{Re}(s)>\frac{1}{2}, s \notin\left(\frac{1}{2}, 1\right]$.

By repeating the arguments of [2, pp. 35-43] almost word for word 
applied to our generalized Eisenstein series, using Proposition 3-3 and the fact that $E(z, s \mid u, v)$ is an automorphic function with respect to $\Gamma^{\prime}$, we see that the following result holds:

Proposition 3-4: $\phi(s \mid u, v)$ may be analytically continued to a meromorphic function in the entire region $\operatorname{Re}(s)>\frac{1}{2}$.

Using Proposition 3-4, we may complete the proof of Theorem 3-1 by making the following observation: The terms of the series for $\phi(s \mid 0,0)$ dominate the absolute values of the terms of the series for $\phi(s \mid u, v)$ for $s>1$. However, since $\phi(s \mid 0,0)$ has a simple pole at $s=1$, we know that $(s-1) \phi(s \mid u, v)$ is bounded in a region of the form $\{s|| s-1 \mid<\varepsilon, \operatorname{Re}(s)>1\}$. Thus, since $\phi(s \mid u, v)$ meromorphic at $s=1$, we see that $\phi(s \mid u, v)$ has at most a simple pole at $s=1$. This completes the proof of Theorem 3-1.

Remarks: (1) In the case $\Gamma=S L(2, Z)$, the function $\phi(s \mid u, v)$ is holomorphic for $u, v$ not both integers, as is seen from the classical second limit formula and the relation $(* *)$.

(2) It is not generally true that $\phi(s \mid u, v)$ is holomorphic for $u, v$ not both integers. Indeed, if $u, v$ both have denominator dividing $f$ and if $\Gamma=\Gamma(f)$, then

$$
\phi(s \mid u, v)=e^{2 \pi i v} \phi(s \mid 0,0)
$$

so that $\phi(s \mid u, v)$ has a simple pole at $s=1$.

(3) It would be interesting to remove the hypothesis $u$, $v$ rational from Theorem 3-1. We have been able to prove Proposition 3-3 without the assumption. But the proof of Proposition 3-4, as derived from [2, pp. 35-43], does not extend in any obvious way.

\section{Kronecker's Second Limit Formula}

In this section, we will derive our generalization of Kronecker's second limit formula. We will assume throughout that $-1 \in \Gamma, \Gamma \subseteq$ $S L(2, Z)$ and $u$ and $v$ are rational. Then, by the results of Section 3, we may write

$$
\phi(s \mid u, v)=\frac{A(u, v)}{s-1}+B(u, v)+\cdots
$$


in a neighborhood of $s=1$.

By letting $s$ tend to 1 from the right in (6), we see that

$$
\begin{array}{r}
\lim _{s \rightarrow 1^{+}}\left[E\left(\sigma_{\infty} z, s \mid u, v\right)-\sqrt{\pi} y^{1-s} \frac{\Gamma\left(s-\frac{1}{2}\right)}{\Gamma(s)} \phi(s \mid u, v)\right] \\
=y e^{2 \pi i v}+2 \pi \sqrt{y} \sum_{\substack{m=-\infty \\
m \neq 0}}^{\infty} b_{m}(z \mid u, v) e^{2 \pi i m x},
\end{array}
$$

where

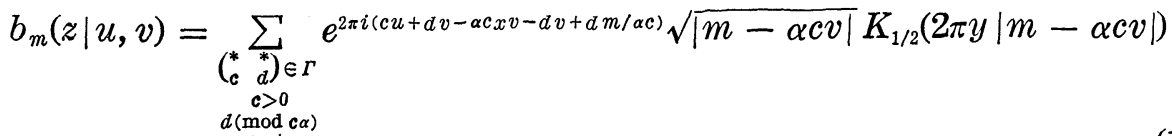

$$
\begin{aligned}
& d(\bmod c \alpha) \\
& =\frac{1}{2} y^{-1 / 2} \sum_{\substack{\left.\left(\begin{array}{c}
* \\
c \\
d
\end{array}\right) \in \Gamma \\
d \bmod c \alpha\right) \\
c>0 \\
\alpha c v \neq m}} e^{2 \pi i(c u-\alpha c v x+d m / \alpha c)} e^{-2 \pi y|m-\alpha c v|} .
\end{aligned}
$$

Let us rewrite the double sum implied in (12) somewhat. Let us sum over all $c$ from 1 to $\infty$ and then sum over $d(\bmod c \alpha)$ for which $(c, d)$ is the second row of an element of $\Gamma$, making the convention that an empty summation equals 0 . Then (12) may be rewritten

$$
\begin{aligned}
& \lim _{s \rightarrow 1_{+}}\left[E\left(\sigma_{\infty} z, s \mid u, v\right)-\sqrt{\pi} y^{1-s} \frac{\Gamma\left(s-\frac{1}{2}\right)}{\Gamma(s)} \phi(s \mid u, v)\right]
\end{aligned}
$$

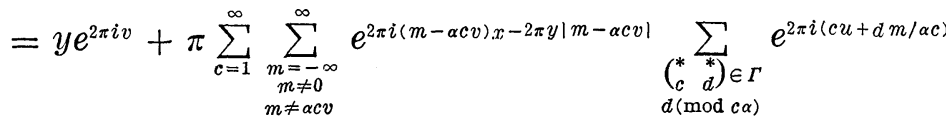

$$
\begin{aligned}
& =y e^{2 \pi i v}+\pi \sum_{c=1}^{\infty} \sum_{\substack{m-\alpha c v>0 \\
m \neq 0}} e^{2 \pi i(m-\alpha c v) z} \sum_{\substack{* \\
\left(\begin{array}{c}
* \\
d \\
d
\end{array}\right) \in T \\
d(\bmod c \alpha)}} e^{2 \pi i(c u+d m / \alpha c)} \\
& +\pi \sum_{c=1}^{\infty} \sum_{\substack{m-\alpha c v>0 \\
m \neq 0}} e^{2 \pi i(m-\alpha c v) \bar{z}} \sum_{\substack{\left(\begin{array}{c}
* \\
c \\
d(\operatorname{lod}) \\
d(\bmod c \alpha)
\end{array} \\
m=\Gamma\right.}} e^{2 \pi i(c u+d m / \alpha c)} .
\end{aligned}
$$

However, the last summand may be rewritten as follows:

$$
\begin{aligned}
& \pi \sum_{c=1}^{\infty} \sum_{\substack{m+\alpha c v>0 \\
m \neq 0}} e^{-2 \pi i(m+\alpha c v) \bar{z}} \sum_{\substack{\left(\begin{array}{c}
* \\
c \\
d(\bmod c \alpha)
\end{array} \\
d(\operatorname{mos}) \in \Gamma\right.}} e^{2 \pi i(c u-d m / \alpha c)}
\end{aligned}
$$

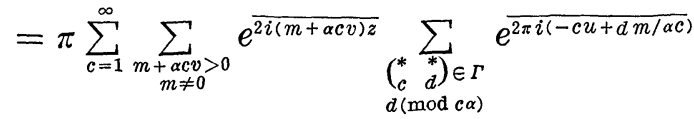


so that

$$
\begin{aligned}
& \lim _{s \rightarrow 1^{+}}\left[E\left(\sigma_{\infty} z, s \mid u, v\right)-\sqrt{\pi} y^{1-s} \frac{\Gamma\left(s-\frac{1}{2}\right)}{\Gamma(s)} \phi(s \mid u, v)\right]
\end{aligned}
$$

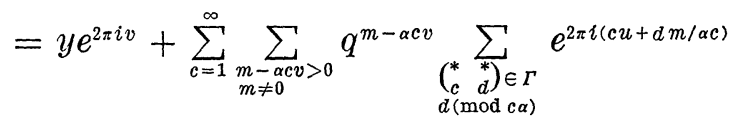

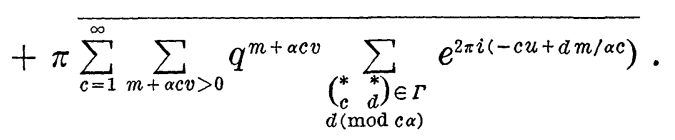

In order to bring (14) into a more convenient form, notice that since $-1 \in \Gamma$, we have

$$
\begin{gathered}
E(z, s \mid u, v)=E(z, s \mid-u,-v), \\
\phi(s \mid u, v)=\phi(s \mid-u,-v) .
\end{gathered}
$$

Therefore, by adding (14) for $(u, v)$ to (14) for $(-u,-v)$, we see that

$$
\begin{aligned}
& 2 \lim _{s \rightarrow 1^{+}}\left[E\left(\sigma_{\infty} z, s \mid u, v\right)-\sqrt{\pi} y^{1-s} \frac{\Gamma\left(s-\frac{1}{2}\right)}{\Gamma(s)} \phi(s \mid u, v)\right] \\
& =2 y \cos (2 \pi v)+\pi \sum_{c=1}^{\infty} \sum_{\substack{m-\alpha c v>0 \\
m \neq 0}} q^{m-\alpha c v} \lambda_{m}(c, u)+\pi \overline{\sum_{c=1}^{\infty} \sum_{\substack{m-\alpha c v>0 \\
m \neq 0}} q^{m-\alpha c v} \lambda_{m}(c, u)} \\
& +\pi \sum_{c=1}^{\infty} \sum_{\substack{m+\alpha c v>0 \\
m \neq 0}} q^{m+\alpha c v} \lambda_{m}(c, u)+\pi \overline{\sum_{c=1}^{\infty} \sum_{\substack{m+\alpha c v>0 \\
m \neq 0}} q^{m+c v} \lambda_{m}(c, u)},
\end{aligned}
$$

where

$$
\lambda_{m}(c, u)=\sum_{\substack{d \\
\left(\begin{array}{c}
* \\
c \\
c>0 \\
c>0 \\
d(\bmod c \alpha)
\end{array}\right.}} e^{2 \pi i(c u+d m / \alpha c)}
$$

Note now that

$$
2 y \cos (2 \pi v)=\frac{z}{i} \cos (2 \pi v)+\overline{\left(\frac{z}{i} \cos (2 \pi v)\right)} .
$$

Let

$$
A=\pi / \operatorname{vol}(\boldsymbol{H} / \Gamma)=\pi / \iint_{D} \frac{d x d y}{y^{2}}
$$

Then, we have proved [1, Theorem 3-3] that $A=A(0,0)$. Then let us 
rewrite (15) as follows:

$$
\begin{aligned}
\lim _{s \rightarrow 1+}\left[\frac{1}{2 \pi} E\left(\sigma_{\infty} z, s \mid u, v\right)-\frac{1}{2 \sqrt{\pi}} y^{1-s} \frac{\Gamma\left(s-\frac{1}{2}\right)}{\Gamma(s)} \phi(s \mid u, v)\right] \\
=-A\left[-\frac{z}{4 \pi i A} \cos (2 \pi v)-\frac{1}{4 A} \sum_{c=1}^{\infty} \sum_{\substack{\text { cocv>0 } \\
m \neq 0}} q^{m-\alpha c v} \lambda_{m}(c, u)\right. \\
\left.-\frac{1}{4 A} \sum_{c=1}^{\infty} \sum_{\substack{m+\alpha c v>0 \\
m \neq 0}} q^{m+\alpha c v} \lambda_{m}(c, u)\right] \\
-A\left[-\left(\frac{z}{4 \pi i A} / \cos (2 \pi v)\right)-\frac{1}{4 A} \sum_{c=1}^{\infty} \sum_{\substack{m-\alpha c v>0 \\
m \neq 0}} \overline{q^{m-\alpha c v} \lambda_{m}(c, u)}\right. \\
\left.-\frac{1}{4 A} \sum_{c=1}^{\infty} \sum_{\substack{m-\alpha c v>0 \\
m \neq 0}} \overline{q^{m+\alpha c v} \lambda_{m}(c, u)}\right] .
\end{aligned}
$$

This last formula suggests defining the generalized $\eta$-function $\eta_{\Gamma}(z \mid u, v)$

$$
\begin{gathered}
\log \eta_{\Gamma}(z \mid u, v)=-\frac{z}{4 \pi i A} \cos (2 \pi v)-\frac{1}{4 A} \sum_{c=1}^{\infty} \sum_{\substack{m-\alpha c v>0 \\
m \neq 0}} q^{m-\alpha c v} \lambda_{m}(c, u) \\
-\frac{1}{4 A} \sum_{c=1}^{\infty} \sum_{\substack{m+\alpha c v>0 \\
m \neq 0}} q^{m+\alpha c v} \lambda_{m}(c, u) .
\end{gathered}
$$

Then our limit formula may be written

$$
\begin{gathered}
\lim _{s \rightarrow 1+}\left[\frac{1}{2 \pi} E\left(\sigma_{\infty} z, s \mid u, v\right)-\frac{1}{2 \sqrt{\pi}} y^{1-s} \frac{\Gamma\left(s-\frac{1}{2}\right)}{\Gamma(s)} \phi(s \mid u, v)\right] \\
=-A \log \left|\eta_{\Gamma}(z \mid u, v)\right|^{2} .
\end{gathered}
$$

The reason for inserting the normalizing factor $A$ is to bring our limit formula into agreement with the known classical cases (e.g. $u=v=0$, $\Gamma=S L(2, Z))$.

Let us now bring our limit formula into final form. About $s=1$, we have the expansions

$$
\begin{aligned}
y^{1-s} & =1+(s-1) \log \left(y^{-1}\right)+\cdots \\
\frac{\Gamma\left(s-\frac{1}{2}\right)}{\Gamma(s)} & =\sqrt{\pi}(1-(2 \log 2)(s-1)+\cdots) .
\end{aligned}
$$

Therefore, by (11), we see that in a neighborhood of $s=1$ that

$$
\frac{y^{1-s}}{2 \sqrt{\pi}} \frac{\Gamma\left(s-\frac{1}{2}\right)}{\Gamma(s)} \phi(s \mid u, v)=\frac{C(u, v)}{s-1}+D(u, v)+\cdots,
$$


where

$$
\begin{aligned}
& C(u, v)=\frac{1}{2} A(u, v) \\
& D(u, v)=\frac{1}{2}\left\{B(u, v)+A(u, v) \log \left(y^{-1}\right)-(2 \log 2) A(u, v)\right\}
\end{aligned}
$$

Thus, from (17) we deduce

THEOREM 4-1 (Kronecker's Second Limit Formula): Assume that $\Gamma \subseteq S L(2, Z)$ is a Fuchsian group of the first kind having infinity as a cusp. Further, assume that $\left(\begin{array}{cc}-1 & 0 \\ 0 & -1\end{array}\right) \in \Gamma$. Further, let $u, v \in Q$. Then we have the following limit formula:

$$
\begin{aligned}
\lim _{s \rightarrow 1+} \mid & \frac{1}{2 \pi} E\left(\sigma_{\infty} z, s \mid u, v\right)-\frac{1}{2} A(u, v) \frac{1}{s-1} \mid \\
& =\frac{1}{2} B(u, v)-A(u, v) \log 2-A(u, v) \log (\sqrt{y})-A \log \left|\eta_{\Gamma}(z \mid u, v)\right|^{2},
\end{aligned}
$$

where

$$
\begin{aligned}
& \log \eta_{\Gamma}(z \mid u, v)=-\frac{z}{4 \pi i A} \cos (2 \pi v)-\frac{1}{4 A} \sum_{c=1}^{\infty} \sum_{\substack{m-\alpha c v>0 \\
m \neq 0}} q^{m-\alpha c v} \lambda_{m}(c, u) \\
& -\frac{1}{4 A} \sum_{c=1}^{\infty} \sum_{\substack{m+\alpha c v>0 \\
m \neq 0}} q^{m+\alpha c v} \lambda_{m}(c, u), \\
& \lambda_{m}(c, u)=\sum_{\substack{d \\
\left(\begin{array}{c}
* \\
c \\
d \\
d c>0 \\
d(\bmod c \alpha)
\end{array}\right.}} e^{2 \pi i(c u+d m / \alpha c)} \cdot
\end{aligned}
$$

In case $\Gamma=S L(2, Z), u=v=0$, Theorem $4-1$ is precisely Kronecker's first limit formula. In case $\Gamma$ is arbitrary and $u=v=0$, Theorem 4-1 gives the generalization of Kronecker's limit formula given in [1]. In all other cases, the formula appears to be new.

ConJECTURE: Although the hypotheses $\Gamma \subseteq S L(2, Z), u, v \in \boldsymbol{Q}$ are essential to our method of proof, we strongly suspect that Theorem 4-1 remains true even without them.

\section{The Generalized $\eta$-Function and Dedekind Sums}

It is clear from the manner in which we defined $\eta_{\Gamma}(z \mid u, v)$ that $\eta_{\Gamma}(z \mid u, v)$ is analytic for $z \in H$ and that $\eta_{\Gamma}(z \mid u, v) \neq 0$ for $z \in H$. Let us derive a few elementary properties of this function. Throughout this 
section, we will use that branch of the log function whose argument runs from 0 (included) to $2 \pi$ (excluded). (Note that which branch of $\log$ is used to define $\eta_{\Gamma}(z \mid u, v)$ is immaterial.)

Since $E(z, s \mid u, v)$ satisfies the transformation property (2), we see that $E\left(\sigma_{\infty} z, s \mid u, v\right)$ satisfies:

$$
E\left(\sigma_{\infty} z, s \mid a u+b v, c u+d v\right)=E\left(\sigma_{\infty} z, s \mid u, v\right)
$$

for $\sigma=\sigma_{\infty}^{-1}\left(\begin{array}{ll}a & b \\ c & d\end{array}\right) \sigma_{\infty} \in \sigma_{\infty}^{-1} \Gamma \sigma_{\infty}$. Therefore, by reasoning precisely as in [1, Theorem 3-2], we deduce a transformation property for $\log \eta_{\Gamma}(z \mid u, v)$.

Let us fix $\sigma=\sigma_{\infty}^{-1}\left(\begin{array}{ll}a & b \\ c & d\end{array}\right) \sigma_{\infty} \in \sigma_{\infty}^{-1} \Gamma \sigma_{\infty}$. Then by (18), both of the functions $E\left(\sigma_{\infty} \sigma z, s \mid a u+b v, c u+d v\right)$ and $E\left(\sigma_{\infty} z, s \mid u, v\right)$ have the same residue at $s=1$. On the other hand, by Theorem $3-1$, these residues are, respectively, $A(a u+b v, c u+d v)$ and $A(u, v)$. Thus,

$$
A(a u+b v, c u+d v)=A(u, v) \quad\left(\sigma=\left(\begin{array}{ll}
a & b \\
c & d
\end{array}\right) \in \Gamma\right) .
$$

Another way of putting (19) is as follows:

THEOREM 5-1: Let $\Gamma$ act on $\boldsymbol{Q} \times \boldsymbol{Q}$ by the formula

$$
\sigma \cdot(u, v)=(a u+b v, c u+d v), \quad \sigma=\left(\begin{array}{ll}
a & b \\
c & d
\end{array}\right) \in \Gamma, \quad(u, v) \in \boldsymbol{Q} \times \boldsymbol{Q} .
$$

Then the residue of $\phi(s \mid u, v)$ is constant on the orbits of $\boldsymbol{Q} \times \boldsymbol{Q}$ under $\Gamma$.

For example, if $\Gamma=S L(2, Z)$, then there are two orbits: $\{(0,0)\}$ and $\boldsymbol{Q} \times \boldsymbol{Q}-\{(0,0)\}$. On the former, $\phi(s \mid u, v)$ has a simple pole with residue $A$. On the latter $\phi(s \mid u, v)$ is regular, as we see from the classical Kronecker limit formula.

It turns out that the behavior of $\log _{\eta}(z \mid u, v)$ is quite different if $A(u, v)$ is zero or non-zero. Therefore, let us make the following definition:

Definition 5-2: Let $(u, v) \in \boldsymbol{Q} \times \boldsymbol{Q}$. We say that $(u, v)$ is regular or irregular according to whether $A(u, v)$ is zero or not. By Theorem 5-1 the orbits of $\boldsymbol{Q} \times \boldsymbol{Q}$ under $\Gamma$ consist of either all regular or all irregular points. Thus, we will speak of regular or irregular orbits. 
Let us subtract the pole term from both sides of (18) (taking into account (19)) and let us pass to the limit $s=1$. Then by Theorem 3-1 and (19), we see that

$$
\begin{gathered}
-A(u, v) \log (\sqrt{y(\sigma z)})-A \log \left|\eta_{\Gamma}(\sigma z \mid a u+b v, c u+d v)\right|^{2} \\
=-A(u, v) \log (\sqrt{y})-A \log \left|\eta_{\Gamma}(z \mid u, v)\right|^{2}
\end{gathered}
$$

for $\sigma=\sigma_{\infty}^{-1}\left(\begin{array}{ll}a & b \\ c & d\end{array}\right) \sigma_{\infty} \in \sigma_{\infty}^{-1} \Gamma \sigma_{\infty}$. However, since $y(\sigma z)=y /|c \alpha z+d|^{2}$, we see that

$$
\begin{gathered}
A\left[\log \left|\eta_{\Gamma}(\sigma z \mid a u+b v, c u+d v)\right|-\log \left|\eta_{\Gamma}(z \mid u, v)\right|\right] \\
=\frac{1}{2} A(u, v) \log |c \alpha z+d| .
\end{gathered}
$$

Note that from (21), it is clear that $A(u, v)$ is always real. Moreover, from (21), it is also clear that there exists a real number $S_{\Gamma}(\sigma \mid u, v)$, not depending on $z$, such that

$$
\begin{aligned}
& \log \eta_{\Gamma}(\sigma z \mid a u+b v, c u+d v)-\log \eta_{\Gamma}(z \mid u, v) \\
& \quad=\frac{1}{2} \frac{A(u, v)}{A} \log (c z+d)+\pi i S_{\Gamma}(\sigma \mid u, v) .
\end{aligned}
$$

Thus, finally, we deduced

THEOREM 5-3: Let $\sigma=\sigma_{\infty}^{-1}\left(\begin{array}{ll}a & b \\ c & d\end{array}\right) \sigma_{\infty}$ and let $(u, v) \in \boldsymbol{Q} \times \boldsymbol{Q}$.

(1) If $(u, v)$ belongs to an irregular orbit, then

$$
\begin{aligned}
& \log \eta_{\Gamma}(\sigma z \mid a u+b v, c u+d v) \\
& \quad=\log \eta_{\Gamma}(z \mid u, v)+\frac{1}{2} \frac{A(u, v)}{A} \log (c z+d)+\pi i S_{\Gamma}(\sigma \mid u, v)
\end{aligned}
$$

with $A(u, v) \neq 0$.

(2) If $(u, v)$ belongs to a regular orbit, then

$$
\log \eta_{\Gamma}(\sigma z \mid a u+b v, c u+d v)=\log \eta_{\Gamma}(z \mid u, v)+\pi i S_{\Gamma}(\sigma \mid u, v) .
$$

The quantities $S_{\Gamma}(\sigma \mid u, v)$ are generalizations of the classical Dedekind sums.

Let us close this paper with a comment which generalizes an observation of Siegel [3]. Suppose that $(u, v)$ belongs to a regular orbit and suppose that $f$ is a common denominator for $u$ and $v$. Let $\Gamma^{\prime}=\Gamma \cap \Gamma(f)$. Then $\Gamma^{\prime}$ is a subgroup of finite index in $\Gamma$. Moreover, from the defini- 
tion of $\Gamma(f)$, we see that if $\sigma_{1}=\left(\begin{array}{ll}a & b \\ c & d\end{array}\right) \in \Gamma(f)$, then

$$
\log \eta_{\Gamma}(z \mid a u+b v, c u+d v)=\log \eta_{\Gamma}(z \mid u, v) .
$$

Therefore, we derive from Theorem 5-3 that if $\sigma=\sigma_{\infty}^{-1}\left(\begin{array}{ll}a & b \\ c & d\end{array}\right) \sigma_{\infty} \in \sigma_{\infty}^{-1} \Gamma^{\prime} \sigma_{\infty}$, then

$$
\log \eta_{\Gamma}(\sigma z \mid u, v)=\log \eta_{\Gamma}(z \mid u, v)+\pi i S_{\Gamma}(\sigma \mid u, v) .
$$

In other words, $\log \eta_{\Gamma}(z \mid u, v)$ is an abelian integral for the group $\sigma_{\infty}^{-1} \Gamma^{\prime} \sigma_{\infty}$ and the Dedekind sum appears as a period of this abelian integral. Siegel observed this fact for $\Gamma=S L(2, Z),(u, v) \neq(0,0)$.

\section{BIBLIOGRAPHY}

[1] Goldstein, L. Dedekind Sums for a Fuchsian Group, I, Nagoya Math. J. 50 (1973), 21-47.

[2] Kubota, T. Elementary Theory of Eisenstein Series, Kodansha Press, Tokyo, 1973.

[ 3 ] Siegel, C. L. Advanced Analytic Number Theory, Tata Institute of Fundamental Research, Bombay, 1961.

University of Maryland

Department of Mathematics 